\title{
Up-regulation of RNF187 induces hepatocellular carcinoma cell epithelial to mesenchymal transitions
}

\author{
Song-Lin $\mathbf{Y u}^{1, *}$, Jin-Cai Wu ${ }^{2, *}$, Peng-Fei Liu ${ }^{1}$, Kai Liu ${ }^{1}$, Chun $\mathrm{Ye}^{1}$, Kai-Lun Zhou ${ }^{2}$, \\ Zhuo-Ri $\mathbf{L i}^{2}$ and $\mathrm{Ya}$ a-Ping $X \mathbf{u}^{3}$ \\ ${ }^{1}$ Department of General Surgery, Tongji Hospital, Tongji University School of Medicine, Shanghai 200065, China \\ ${ }^{2}$ Department of Hepatobiliary Surgery and Organ Transplantation, Hainan Provincial People's Hospital, University of South \\ China, Haikou 570311, China \\ ${ }^{3}$ Department of Gastroenterology Shanghai Tenth People's Hospital, Tongji University School of Medicine, Shanghai 200072, \\ China \\ *These authors contributed equally to this work
}

Correspondence to: Ya-Ping Xu, email: xyp_0115xu@126.com

Zhuo-Ri Li, email: lizhuori_2010wu@163.com

Keywords: hepatocellular carcinoma, RNF187, EMT, prognosis

Received: April 21, $2017 \quad$ Accepted: September 23, 2017

Published: October 19, 2017

Copyright: Yu et al. This is an open-access article distributed under the terms of the Creative Commons Attribution License 3.0 (CC BY 3.0), which permits unrestricted use, distribution, and reproduction in any medium, provided the original author and source are credited.

\section{ABSTRACT}

Ring finger protein 187 (RNF187) has been identified to be a co-activator linking c Jun to Ras signaling. However, the expression and function of RNF187 in hepatocellular carcinomas (HCC) remains unclear. Here, we tried to determine the expression and roles of RNF187 in hepatocellular carcinomas (HCC). The expression of RNF187 was determined in HCC tissues and cell lines, and we found that RNF187 expressed highly in HCC tissues compared with the corresponding adjacent liver tissues both in mRNA and protein level, which was consistent with the result of immunohistochemistry on HCC tissue microarrays. In HCC cell lines, the level of RNF187 was positively associated with the HCC cells metastatic potential. By the RNF187 interference and cDNA transfection, we showed that the high level of RNF187 induced the HCC cells invasion and metastasis both in vitro and in vivo, as well as the high ability of colony formation. Mechanistically, we detected the high level of RNF187 promoted cell scatter by inducing epithelial-mesenchymal transition (EMT). Clinically, the high level of RNA187 was significantly correlated with a malignant phenotype, including larger tumor size, multiple tumors, and microvascular invasion. Importantly, high level of RNF187 correlated with HCC patients' shorter OS and lower disease free survival rates than those with low level of RNF187. Our results revealed that elevated expression of RNF187 induced hepatocellular carcinoma cell epithelial to mesenchymal transitions, and represented a novel marker for predicting the poor prognosis of HCC.

\section{INTRODUCTION}

Hepatocellular carcinoma (HCC) is the fifth commonest malignancy and the third leading cause of cancer-related deaths in the world [1]. Clinically, $\mathrm{HCC}$ is characterized by its high invasive ability, the high incidence of recurrence, and the finite availability of effective therapy [2]. To date, surgery is still the preferred treatment of $\mathrm{HCC}$, but unfortunately multifocal progression and early distant metastases hinder surgical curative treatment in most $\mathrm{HCC}$ patients $[3,4]$. Thus, an understanding of the molecular mechanisms underlying $\mathrm{HCC}$ metastasis and recurrence is urgently required for effective chemotherapy treatment of HCC patients.

Substantial research, that focused on tumor development, has revealed that the epithelialmesenchymal transition (EMT) is an essential reversible process during tumor progression [5]. Initially, EMT 
is found to be a fundamental procedure involved in embryonic development during which epithelial cells lose their differentiated properties and gain mesenchymal characteristics [6]. Then, a plethora of studies delineated the important role of EMT in pathogenesis of metastasis in epithelial tumors [7]. Indeed, the EMT cell is commonly apparent at the invading margin of the tumor mass and is likely to mediate cellular detachment and eventual metastasis [8]. Moreover, induction of EMT in cancer cell lines with low metastasis potential results in the acquisition of high metastatic properties in vitro $[9,10]$. Of note is that several new studies have further demonstrated that cancer cells undergoing EMT appeared to gain the ability to resist apoptosis, chemotherapy and immunotherapy, respectively and also acquire stem cell features [11-13], which further emphasizes the role of EMT in mediating cancer metastasis, and the value of uncovering the fundamental molecular mechanisms underlying EMT.

The ubiquitin-proteasome system regulates a wide range of physiological processes including signal transduction, proliferation and apoptosis [14]. The dysregulation of ubiquitination was found to be directly involved in human cancers including $\mathrm{HCC}$ and may function as oncogene or tumor suppressor [15]. For example, the overexpression of ubiquitin ligase E3C promoted HCC progression by regulating tumor cell EMT [16], and a level of ubiquitin-specific protease 7 accelerated p14 ${ }^{\text {ARF }}$ degradation by deubiquitinating thyroid hormone receptorinteracting protein 12 and promoting HCC progression [17]. Ring finger protein 187 (RNF187, also known as RACO1 or RACO-1) is a RING domain-containing ubiquitin E3 ligase. Normally, RNF187 is unstable in unstimulated conditions due to K48-linked autoubiquitination, and is stable in nondegradative K63-linked ubiquitination by the competition of degradative K48-linked ubiquitination regulated by activation of the Ras pathway [18]. Recently, several studies have examined the functions of RNF187. For example, RNF187 depletion was found to reduce cellular proliferation and downregulate several growthassociated AP-1 target genes, such as cyclin-dependent kinase 1 (CDC2), heparin binding EGF like growth factor (HBEGF) and cyclinD1 [19]. Additionally, transgenic overexpression of RNF187 was shown to enhance intestinal tumor formation by inducing aberrant Wnt signaling and through cooperation with oncogenic Ras in colon epithelial hyperproliferation [20]. Although, the reports on RNF187 functions are very limited at present, especially in tumors, the existing data show that it may play an important role in tumorigenesis and development.

Here, we tried to determine the expression of RNF187 in HCC tissues and cell lines. The role of RNF187 in HCC cells was investigated both in vitro and in vivo by using RNF187 interference and cDNA transfection. Finally, the clinical significance of RNF187 expression was further analyzed using tissue microarray (TMA) in 209 patients with HCC.

\section{RESULTS}

\section{The expression of RNF187 is elevated in human $\mathrm{HCC}$ and positively associated with $\mathrm{HCC}$ malignant phenotypes}

Initially, the expression of RNF187 was determined by $q$ RT-PCR and western blotting in $15 \mathrm{HCC}$ and their matched peritumor tissues. The RNF187 expression was found to be higher in HCC than in matched peritumor tissues both at the level of mRNA $(3.45 \pm 0.29$ vs. 1.74 $\pm 0.13, P<0.01$, Figure $1 \mathrm{~A}$ and $1 \mathrm{~B})$ and protein $(2.75$ \pm 0.09 vs. $1.24 \pm 0.02, P<0.01$, Figure $1 \mathrm{C}$ and 1D). Next, we examined the expression of RNF187 by TMAs including 209 patients with HCC (Figure 1E and 1F). Immunohistochemical results revealed that RNF187 was located in the cell cytoplasm and nuclei of neoplastic cells and highly expressed in 94 cases with variable intensities (44.98\%), while low level of RNF187 were found in $55.02 \%$ (low expression, 115/209) tumor tissues.

In $\mathrm{HCC}$ tissues, RNF187 $7^{\text {high }}$ was significantly correlated with microvascular/bile duct invasion $(P=0.003)$, high TNM stage $(P=7.64 \mathrm{E}-11)$, multiple tumor $(P=0.026)$, and large tumor size $(P=1.90 \mathrm{E}-13)$. However, other clinical characteristics including age, sex, HBsAg background, tumor differentiation, liver cirrhosis, preoperative serum alpha-fetoprotein (AFP), and Child-Pugh scores were not significantly related to the expression of RNF187 (Table 1). The above results indicate that high levels of RNF187 may promote HCC progression.

\section{Elevated expression of RNF187 is associated with increased invasion potential of $\mathrm{HCC}$ cells}

Here, we further sought to address the role of RNF187 in HCC cells. As shown in Figure 2A, the HCCLM3, MHCC97-H, Huh7 and PLC/PRF/5 cells expressed high levels of RNF187, while SMMC-7721 and HepG2 cells expressed low levels of RNF187 both in mRNA and protein levels. The immunofluorescent staining also showed that HCCLM3 and Huh7 cells expressed high level of RNF187, and we detected only low levels of RNF187 in PLC/PRF/5 and HepG2 cells (Figure 2B).

Next, we silenced RNF187 in HCCLM3 using specific vshRNAs and elevated RNF187 expression in HepG2 cells by cDNA-RNF187 transfection to investigate the effect of RNF187 on cell invasion and apoptosis. Among the 3 candidate shRNAs tested, shRNA-2 (designated shRNF187) was shown to be most efficient, and was therefore selected for subsequent studies (Figure 2C). By transwell assay, we found that the interference of RNF187 in HCCLM3 cells inhibited the invasion of HCCLM3 cells, while the up-regulation of RNF187 in HepG2 cells enhanced the invasion of HepG2 cells (Figure 2D). Using the wound healing assay, we showed 
that the RNF187 expression in HCC cells was positively associated with the migration of HCC cells (Figure 2D), which concurred with the in vivo examination (Figure $2 \mathrm{~F}$ and $2 \mathrm{G})$. Additionally, we also showed the ability of clone formation was elevated in cells with high level of RNF187 (Figure 2H), and the MTT assay showed that the knockdown of RNF187 expression in HCCLM3 cells significantly inhibited cell activity, while the forced RNF187 expression upregulated the HepG2 cell activity (Figure 2I). These results indicated the high levels of RNF187 was associated with an increased metastatic potential of HCC cells.

\section{High expression of RNF187 induces HCC cell EMT}

To delineate further the molecular basis by which RNF187 overexpression induces the invasion of HCC cells, whole genome transcriptome analysis on HCCLM3control, HCCLM3-shRNF187, HepG2-control and HepG2-RNF187 cells were performed using RNA-seq (Figure 3A). By defining a threshold of the average FPKM $\geq 1$, and the cut-off as more than a 2 fold change, 123 down-regulated genes and 156 up-regulated genes were found to be changed in both groups (Figure 3B), which mainly enriched cell migration, EMT, positive regulation of GTPase activity, actin cytoskeleton organization, positive regulation of $\mathrm{PI} 3 \mathrm{~K}$ signaling, Wnt signal pathway, TGF beta receptor signaling pathway by Gene Ontology (GO) analysis, and Kyoto encyclopedia of genes and genomes (KEGG) analysis also indicated they activate pathways involved in cancer, Ras signaling pathway, tight junction, regulation of actin cytoskeleton, etc (Figure 3C). Here, we further compared the epithelial, mesenchymal marker (Figure 3D), c-Myc and adherent molecules. As shown in Figure $3 \mathrm{E}$, the E-cadherin mRNA was downregulated in cells overexpressing RNF187, while Snail, Vimentin, FN1 and c-Myc were upregulated, which was further validated by western blotting (Figure 3F). Then, we examined the cellular morphology of HCCLM3-control, HCCLM3shRNF187, HepG2-control and HepG2-RNF187 cells, and found a distinct morphological difference was observed between cells expressing high level of RNF187 and low level of RNF187 (HCCLM3-control vs. HCCLM3shRNF187, HepG2-control vs. HepG2-RNF187), and cells expressing a low level of RNF187 presented the typical
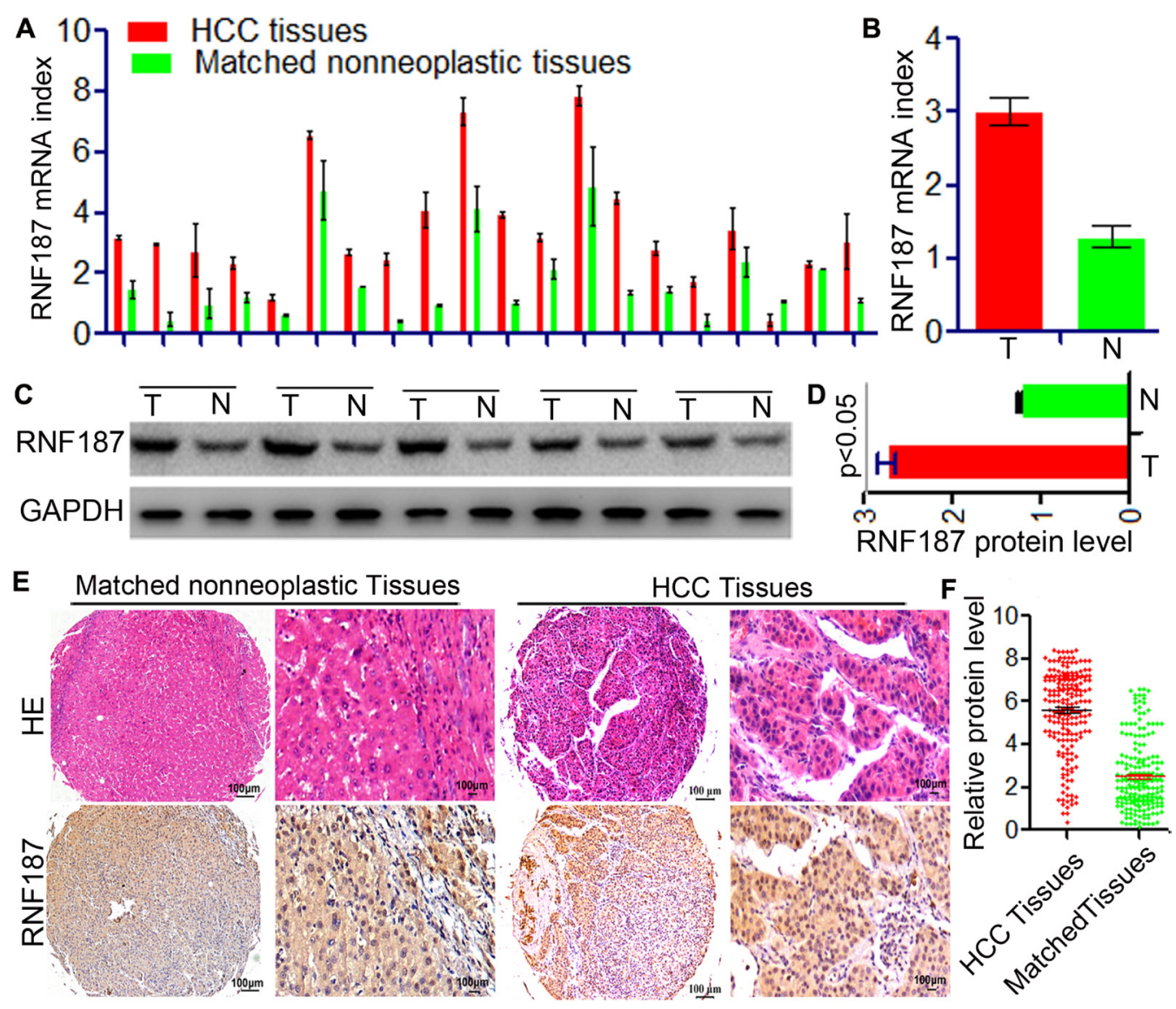

Figure 1: Up-regulation of RNF187 in HCC tissues. (A and B) $q$ RT-PCR showed that RNF187 mRNA level in HCC tissues was higher than that in adjacent non-tumorous liver tissues with statistically significant difference $(P<0.01)$; (C and D) Western blotting showed RNF187 protein expression in HCC adjacent non-tumorous tissues $(P<0.01)$; $(\mathbf{E}$ and $\mathbf{F})$ Immunohistochemical staining demonstrated that expression level of RNF187 protein in HCC tissues was higher than that in adjacent non-tumorous tissues $(P<0.01)$. 
Table 1: Association of RNF187 expression with clinicopathological parameters of HCC patients

\begin{tabular}{|c|c|c|c|c|}
\hline \multirow[t]{2}{*}{ ClinicopathologicalParameters } & \multirow[t]{2}{*}{ Total } & \multicolumn{2}{|c|}{ RNF187 Expression } & \multirow[t]{2}{*}{$p$ value } \\
\hline & & Negative & Positive & \\
\hline & 209 & 115 & 94 & \\
\hline \multicolumn{5}{|l|}{ Sex } \\
\hline Male & 179 & 94 & 85 & 0.055 \\
\hline Female & 30 & 21 & 9 & \\
\hline Age range,yr & & & & 0.403 \\
\hline$\leq 50$ & 93 & 48 & 45 & \\
\hline$>50$ & 116 & 67 & 49 & \\
\hline Tumor numbler & & & & 0.026 \\
\hline 1 & 166 & 98 & 68 & \\
\hline$\geq 2$ & 43 & 17 & 26 & \\
\hline Tumor size, $\mathrm{cm}$ & & & & $1.90 \mathrm{E}-13$ \\
\hline$\leq 5$ & 122 & 93 & 29 & \\
\hline$>5$ & 87 & 22 & 65 & \\
\hline TNM stage & & & & 7.64E-11 \\
\hline I-II & 144 & 101 & 43 & \\
\hline III-IV & 65 & 14 & 51 & \\
\hline Grade of differentiation & & & & 0.065 \\
\hline I-II & 152 & 89 & 63 & \\
\hline III-IV & 57 & 26 & 31 & \\
\hline Microvascular/ bile duct invasion & & & & 0.003 \\
\hline Positive & 59 & 23 & 36 & \\
\hline Negative & 150 & 92 & 58 & \\
\hline HBX & & & & 0.491 \\
\hline Positive & 167 & 94 & 73 & \\
\hline Negative & 42 & 21 & 21 & \\
\hline Cirrhosis & & & & 0.855 \\
\hline Positive & 187 & 104 & 83 & \\
\hline Negative & 22 & 11 & 11 & \\
\hline AFP & & & & 1.000 \\
\hline$\leq 400$ & 131 & 72 & 59 & \\
\hline$>400$ & 78 & 43 & 35 & \\
\hline ALT,U/L & & & & 0.684 \\
\hline$\leq 75$ & 181 & 101 & 8580 & \\
\hline$>75$ & 28 & 14 & 14 & \\
\hline
\end{tabular}

Abbreviations and Note: HCC, hepatocellular carcinoma; AFP, alpha-fetoprotein;TNM, tumor node metastasis; HBsAg, hepatitis B surface antigen; ALT, alanine aminotransferase; NS, not significant; The $\mathrm{X}^{2}$ test was used for comparison between groups. $50 \%$ for RNF 187.

cobblestone-like appearance of normal epithelium, while cells expressing a high level of RNF187 took on a spindlelike, fibroblastic morphology (Figure 3G). Then, we probed these cells with epithelial and mesenchymal markers by immunofluorescent staining. As shown in Figure $3 \mathrm{H}$,
HCCLM3-control and HepG2-RNF187 cells exhibited the typical EMT phenotype, including downregulation of E-cadherin and upregulation of vimentin. The above results collectively indicated a high level of RNF187 contributes to HCC cells invasion by inducing cell EMT. 


\section{Clinical significance of RNF187 in the prognosis of HCC patients}

To explore the association of RNF187 expression in HCC tissues with disease prognosis, patients were dichotomized into RNF $187^{\text {high }}$ (moderate and strong; $n=94$ ) or RNF187 $7^{\text {low }}$ (negative and weak; $\mathrm{n}=115$ ) groups (Figure 4A). Statistically, there was a striking inverse association between RNF187 intensity and overall survival (OS; $P=2.29 \mathrm{E}-4)$ and to a less extent diseasefree survival (DFS; $P=0.007$ ) (Figure $4 \mathrm{~B}$ and $4 \mathrm{C}$ ).

Univariate analysis revealed that tumor diameter, tumor differentiation, tumor numbers, microvascular/ bile duct invasion, TNM stage and RNF187 expression were predictors for OS and DFS (Tables 2 and 3), In a multivariate Cox proportional hazards model, TNM stage and tumor differentiation were independent prognostic factor for OS; vascular invasion,TNM stage and tumor differentiation were independent prognostic factor for DFS. Therefore, RNF187 expression is a valuable predictor for OS in patients with HCC.

\section{DISCUSSION}

Here, we firstly report that the RNF187, a RING domain-containing ubiquitin E3 ligase, has an
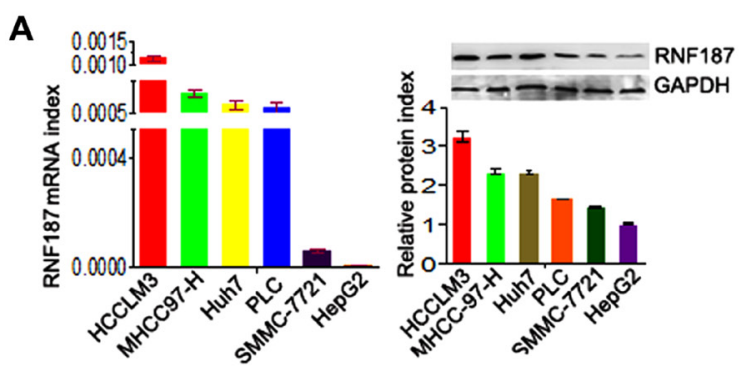

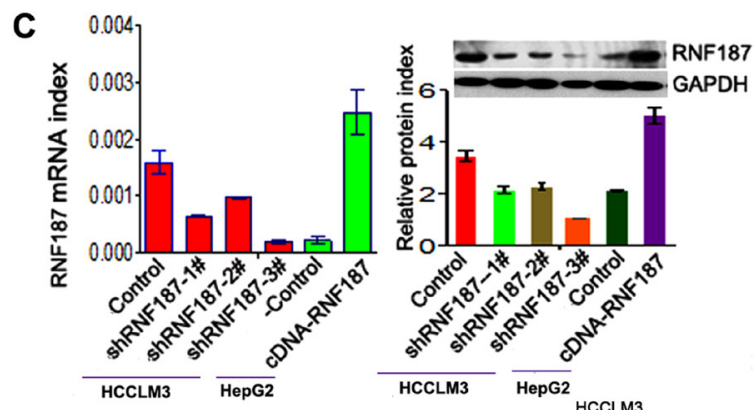

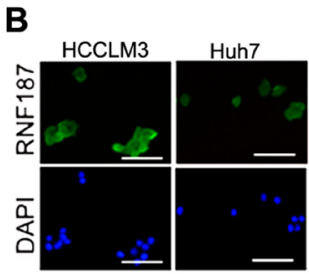
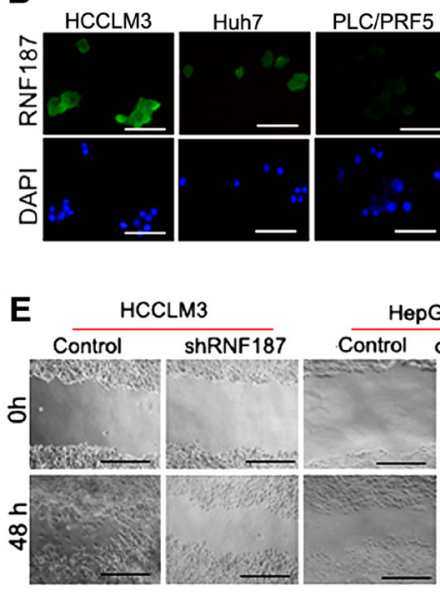

H

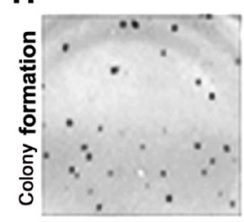

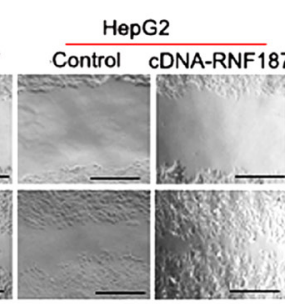

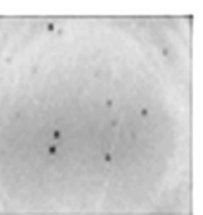

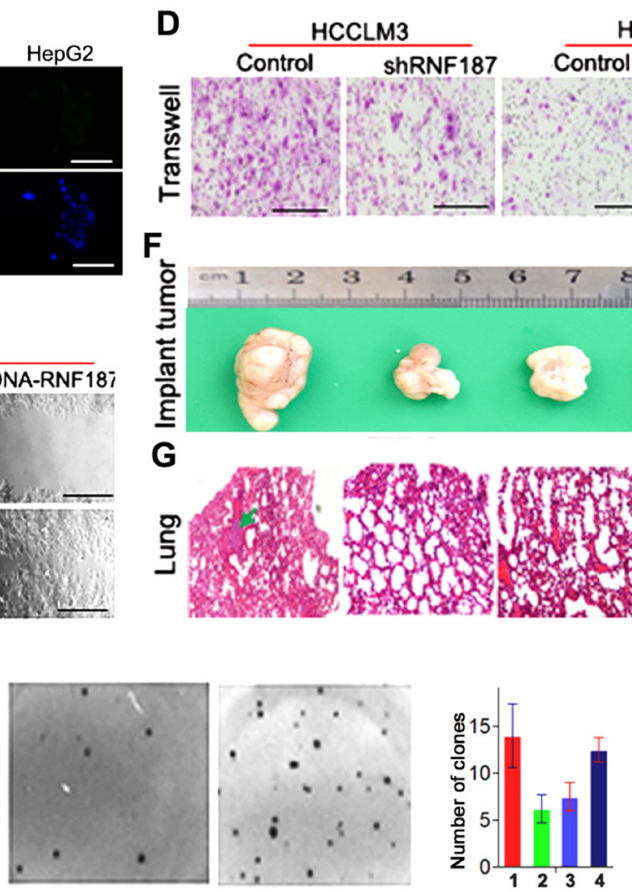
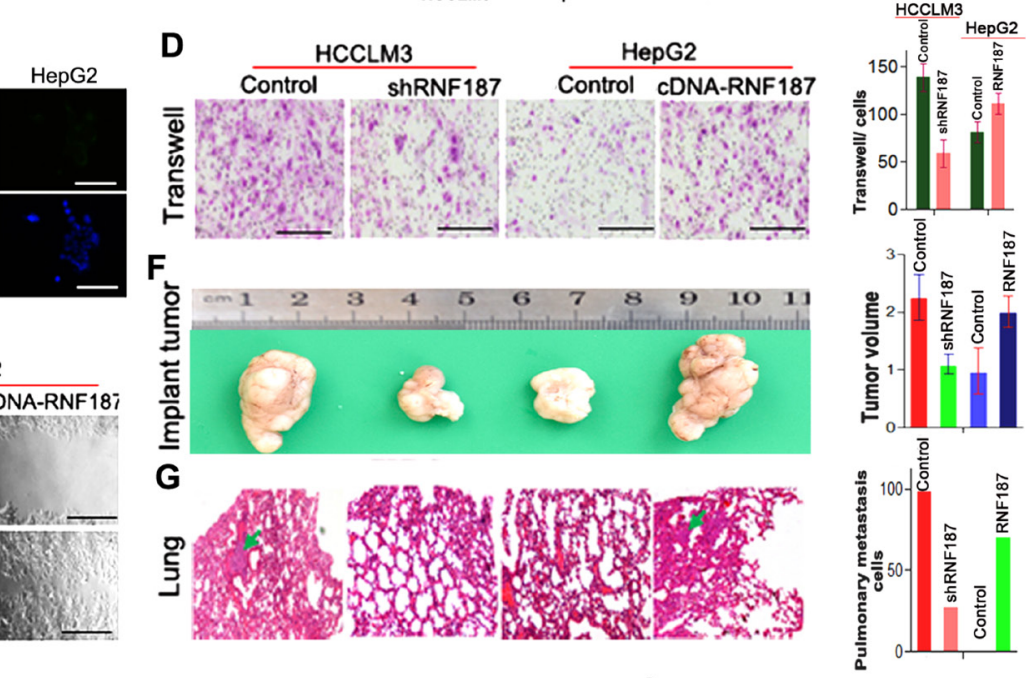

Figure 2: High level of RNA187 promote HCC cells invasion and metastasis both in vitro and in vivo. (A) $q$ RT-PCR showed RNF187 mRNA in HCCLM3, MHCC97-H, Huh7, PLC, SMMC-7721 and HepG2 cell lines; (B) Western blotting showed RNF187 mRNA in HCCLM3, MHCC97-H, Huh7, PLC, SMMC-7721 and HepG2 cell lines; (C) Immunofluorescent staining for RNF187 in HCCLM3, Huh7, PLC and HepG2 cells. DAPI stain (blue) was used to identify nuclei (Scale bar: $200 \mu \mathrm{m}$ ); (D) RNF187 expression was downregulated by pGPU6-GFP-vshRNA-RNF187s in HCCLM3, and up-regulated by pGPU6-RFP-cDNA-RNF187 in HepG2 cells, and \#3 was validated for the most efficient interference of RNF187 by qRT-PCR and immunoblotting. (E) Cells with low RNF187 expression migrated slowly compared with those with high RNF187 expression at $48 \mathrm{~h}$ for wound channel closure. (F) The clone formation was elevated in cells with high level of RNF187. (G and H) Transwell assay showed that the numbers of invading cells in groups with low RNF187 expression were higher than those with high RNF187 expression, and the volume of the tumors derived from HCC isogenic cell lines was calculated in vivo for 6 weeks; Serial sections from mouse lung showed the metastasis ability of cancer cells expressing different RNF187 (Scale bar: $50 \mu \mathrm{m}$ ); and cell proliferation was positively associated with RNF187 expression in vitro. Magnification, $\times 200$ (E and G) and $\times 100$ (I). 
Table 2: Univariate and multivariate analysis of prognostic factors of OS

\begin{tabular}{lcccc}
\hline Variable & \multicolumn{2}{c}{ Univariate } & \multicolumn{2}{c}{ Multivariate } \\
\cline { 2 - 5 } & HR $(\mathbf{9 5 \%} \mathbf{C l})$ & $\boldsymbol{p}$ value & HR (95 \% Cl) & $\boldsymbol{p}$ value \\
\hline RNF187 expresssion (positive vs negative) & $1.766(1.157-2.696)$ & 0.008 & $0.810(0.478-1.375)$ & 0.436 \\
Tumor diameter $(\mathrm{cm})(\leq 5$ vs $>5)$ & $1.725(1.133-2.628)$ & 0.011 & $0.805(0.395-1.409)$ & 0.367 \\
Tumor number $(1$ vs. $\geq 2)$ & $3.018(1.917-4.749)$ & $1.81 \mathrm{E}-6$ & $1.099(0.570-2.121)$ & 0.778 \\
Vascular invasion (positive vs negative) & $2.392(1.555-3.679)$ & $7.21 \mathrm{E}-5$ & $1.453(0.857-2.464)$ & 0.166 \\
AFP $(\mathrm{ng} / \mathrm{ml})(\leq 400$ vs $>400)$ & $1.427(0.906-2.248)$ & 0.125 & & n.a. \\
TNM stage (I-II vs III-IV) & $6.461(4.170-10.01)$ & $6.63 \mathrm{E}-17$ & $4.922(2.759-5.621)$ & $5.63 \mathrm{E}-8$. \\
Tumor differentiation & $5.608(3.625-8.674)$ & $9.43 \mathrm{E}-15$ & $3.478(2.085-5.495)$ & $8.13 \mathrm{E}-7$ \\
Sex (male vs female) & $1.776(0.891-3.544)$ & 0.103 & & n.a. \\
Liver cirrhosis (positive vs negative) & $0.748(0.345-1.620)$ & 0.462 & & n.a. \\
ALT (U/L) $(\leq 50$ vs $>50)$ & $1.003(0.545-1.846)$ & 0.993 & & n.a. \\
Age (years) $(\leq 50$ vs $>50)$ & $1.766(0.891-3.544)$ & 0.103 & & n.a. \\
HBsAg (positive vs negative) & $0.817(0.491-1.358)$ & 0.435 & & n.a. \\
\hline
\end{tabular}

Abbreviations and Note: HCC, hepatocellular carcinoma; AFP, alpha-fetoprotein; TNM, tumor node metastasis; HBsAg, hepatitis B surface antigen; ALT, alanine aminotransferase; n.a., not applicable; The $\mathrm{X}^{2}$ test was used for comparison between groups. $50 \%$ for RNF187.

Table 3: Univariate and multivariate analysis of prognostic factors of DFS

\begin{tabular}{lcccc}
\hline \multirow{2}{*}{ Variable } & \multicolumn{2}{c}{ Univariate } & \multicolumn{2}{c}{ Multivariate } \\
\cline { 2 - 5 } & HR (95\%Cl) & $\boldsymbol{p}$ value & HR (95\% Cl) & $\boldsymbol{p}$ value \\
\hline RNF187 expresssion (positive vs negative) & $2.137(1.404-3.253)$ & $3.97 \mathrm{E}-4$ & $1.142(0.687-1.900)$ & 0.608 \\
Tumor diameter $(\mathrm{cm}, \leq 5$ vs $>5)$ & $1.110(1.055-1.167)$ & $5.24 \mathrm{E}-5$ & $1.252(0.696-2.176)$ & 0.476 \\
Tumor number $(1$ vs. $\geq 2)$ & $1.618(1.100-2.380)$ & 0.015 & $1.251(0.673-2.328)$ & 0.478 \\
Vascular invasion (positive vs negative) & $2.544(1.674-3.867)$ & $1.22 \mathrm{E}-5$ & $1.594(0.883-2.416)$ & 0.025 \\
AFP (ng/ml, $\leq 400$ vs $>$ 400) & $1.151(0.810-1.263)$ & 0.160 & & na \\
TNM stage (I-II vs III-IV) & $4.211(2.756-6.433)$ & $2.94 \mathrm{E}-11$ & $2.332(1.304-4.171)$ & 0.004 \\
Tumor differentiation & $3.273(2.147-4.990)$ & $3.53 \mathrm{E}-8$ & $1.809(1.096-2.985)$ & 0.002 \\
Sex (male $v$ s. female) & $1.441(0.766-2.708)$ & 0.257 & & n.a. \\
Liver cirrhosis (positive vs negative) & $0.677(0.313-1.465)$ & 0.322 & & n.a. \\
ALT (U/L, $\leq 50$ vs $>50)$ & $0.684(0.343-1.362)$ & 0.280 & & n.a. \\
Age (years, $\leq 50$ vs $>50)$ & $1.236(0.809-1.889)$ & 0.327 & & n.a. \\
HBsAg (positive vs negative) & $1.057(0.623-1.791)$ & 0.838 & & n.a. \\
\hline
\end{tabular}

Abbreviations and Note: HCC, hepatocellular carcinoma; AFP, alpha-fetoprotein; TNM, tumor node metastasis; HBsAg, hepatitis B surface antigen; ALT, alanine aminotransferase; n.a., not applicable; The $\mathrm{X}^{2}$ test was used for comparison between groups. $50 \%$ for RNF187.

elevated expression in HCC tissues compared with the corresponding adjacent normal liver tissue both in mRNA and protein levels. In HCC tissues, RNF187 expression exhibits considerable heterogeneity in different samples. Moreover, the high levels of RNF187 were frequently found in tissues of malignant phenotypes, such as microvascular/bile duct invasion, high TNM stage, multiple tumors, and large tumor sizes, which indicates a high level of RNF187 functions as a promoter of HCC. Congruously, we demonstrated in both in vitro and in vivo models that overexpression of RNF187 promotes HCC by inducing HCC cells EMT. Importantly, a high level of RNF187 was found to correlate with poor survival of HCC patients. The above results indicate that high levels of RNF187 promote HCC invasion and metastasis.

Ubiquitination is one of the most abundant protein modifications and is shown to regulate a wide variety of biological processes, such as proteasomal degradation, endocytosis, subcellular localization, and kinase activation [21-23]. Ongoing work revealed that the disruption 


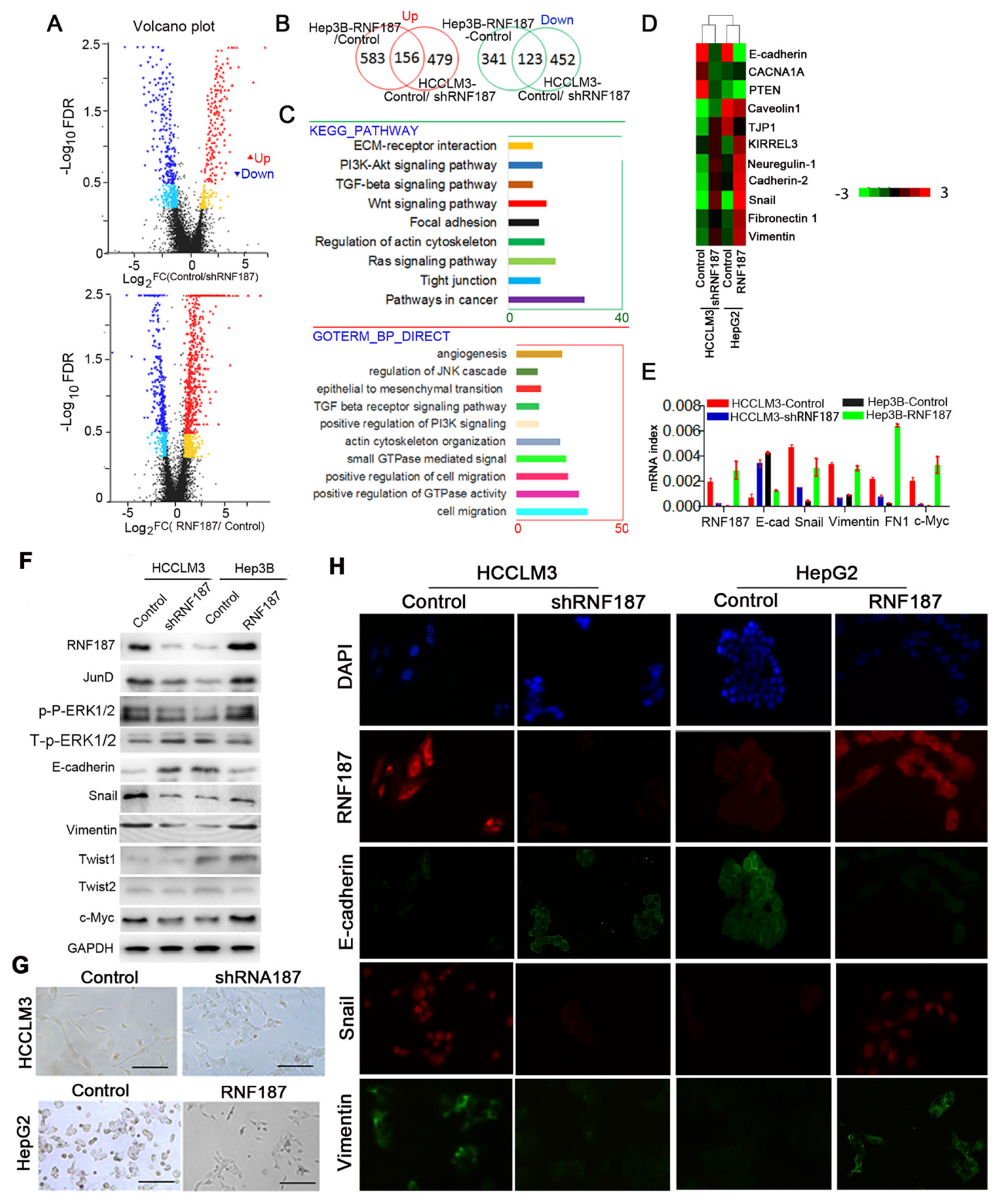

Figure 3: RNF187 overexpression induces an EMT in HCC cells. (A) The RNA-sequence scatter plot showed the up or downregulated gene between Hep3B-Control/ Hep3B-RNF187 cells and HCCLM3-Control/HCCLM3-shRNF187 cells detected by RNASequence; (B) The overlap genes with up or down-regulation between Hep3B-Control/Hep3B-RNF187 cells and HCCLM3-Control/ HCCLM3-shRNF187 cells; (C) The high level of RNF187 in HCC cells majorly involved in the regulation of signaling pathway related to cancer, such as "Pathway in cancer", 'MAPK signaling pathway', 'epithelial-mesenchymal transition', 'Wnt signaling pathway', and "Tight junction"; (D) The hotmap of RNA-seq showed the up-regulation and down-regulation of gene related to EMT; (E) Different expression of the epithelial and mesenchymal markers, as well as the transcription factors and c-Myc were showed between high and low expression RNF187 cancer cells (HepG2-control vs. HepG2-RNF187 and HCCLM3-control vs. HCCLM3-vshRNF187 cells; (F) Western blotting determined RNF187, E-cadherin, FN1, Vimentin, Snail, Twist1, Twist2, and c-Myc proteins expressiom in HCC cells with different TNF187 expression; (G) Phase-contrast microscopy showed the shapes of HepG2-Mock, HepG2-RNF187, HCCLM3-Mock and HCCLM3-vshRNF187. DAPI stain (blue) was used to identify nuclei (Scale bar: $200 \mu \mathrm{m}$ ); (H) Immunofluorescent staining for RNF187, E-cadherin, Vimentin and Snail in HepG2-mock, HepG2-RNF187, HCCLM3-mock and HCCLM3-shRNF187. DAPI stain (blue) was used to identify nuclei (Scale bar: $200 \mu \mathrm{m}$ ). 
of ubiquitin pathways leads to the development of human diseases, including many types of tumors [24]. For example, many RING finger E3s, including the anaphase promoting complex (APC), MDM2 protooncogene (MDM2), breast cancer associated gene 1 (BRCA1), calcineurin B-like proteins (CBLs), and von Hippel-Lindau tumor suppressor (VHL), have been demonstrated to be implicated in either the suppression or the progression of cancer $[25,26]$. Here, we have shown that RNF187 overexpression functions as a promoter in HCC invasion and metastasis, which is supported by the following evidence. Frist, a high level of RNF187 was positively associated with the metastatic potential of $\mathrm{HCC}$ cells. Second, a high level of RNF187 induces HCC cell EMT. Third, data from HCC patients demonstrated that overexpression of the RNF187 protein was significantly correlated with malignant phenotypes including larger tumor sizes, multiple tumors, and microvascular invasion, which are well established poor prognostic parameters.. Finally, clinical data demonstrated that HCC patients with RNF187 overexpression had poorer OS. In fact, using a modified yeast two-hybrid screen, RNF187 was originally found to be a novel JunD co-activator that links growth factor and oncogenic Ras signalling to the activation of AP-1 [27]. In view of the acknowledged proto-oncoprotein of JunD [28, 29], it is not unreasonable to draw the conclusion that a high level of RNF187 is a promoter of tumor progression.

At present, EMT is believed to be a vital process contributing to the tumor invasiveness and metastatic, including HCC $[30,31]$. Mechanistically, the hyperactivation of PI3K/Akt and Raf/MAPK in EMT have been well demonstrated [8]. For example, Cryab overexpression fosters tumor progression in $\mathrm{HCC}$ by inducing cells EMT through activating the ERK signaling [32]. Here, we also detected an alteration in MAPK signal pathway associated with upregulation or downregulation of RNF187 by RNA-sequence. In HCC tissues, the positive immunostaining of ERK1/2 phosphorylation was increased by up to $45 \%$ [33], which is nearly in lines with the high level of RNF187 detected by immunohistochemistry in our study. The explanation might be due to the MEK/ERK pathway strongly promoting Lys 63-linked ubiquitylation of RNF187, which antagonized Lys 48-linked degradative auto-ubiquitylation of the same Lys residues [18]. In addition, we found that the forced expression of RNF187


Figure 4: RNF187 overexpression is associated with the poorer survival of HCC patients. (A) The representing of expression of RNF187 in HCC tissues (Scale bar $=200 \mu \mathrm{m})$; (B and C) RNF187 overexpression correlates the poorer survival and lower DFS rate of HCC patients. 
in HCC cells could upregulate the level of c-Myc, which has been reported to be a powerful inducer of tumor cell EMT $[6,7]$. In brief, high level of RNF187 promote HCC progression by inducing cell EMT, which closely related to the MAPK signal pathway.

In summary, our results collectively characterize overexpression of RNF187 as a major contributor to the invasion-prone phenotype of HCC, and define RNF1871 as a chemotherapy target for HCCs.

\section{MATERIALS AND METHODS}

\section{Cell lines and plasmids}

$\mathrm{HCC}$ cell lines with different metastatic potentials (HCCLM3, MHCC97-H, Huh7, PLC/PRF/5, SMMC7721 and HepG2) [3] were purchased from the Chinese Academy of Sciences. These cell lines were routinely maintained as the previous report [34].

The vshRNAs were constructed and synthesized by Shanghai Genomeditech Co., Ltd. The target sequence is 5'-TGTGATGGACCGT AGGAAGAATTCAAGAGATTCTTCCTACGGTCCAT CAtttttc- $3^{\prime}$. All transfections were performed as described previously [32].

\section{Patients and specimens}

A total of 209 pathologically confirmed HCC patients, who received curative resection in Hainan Provincial People's Hospital, University of South China and Tongji Hospital, Tongji University between January 2002 and December 2010 were enrolled in the study. The inclusion criteria and patients standardized follow-up procedures have been previous documented [32]. Primary endpoints were disease free survival (DFS) and overall survival (OS) defined as the time intervals between the date of surgery and first report of tumor recurrence or patient death.

\section{RNA extraction and the quantitative real-time polymerase chain reaction (qRT-PCR)}

HCC cells, HCC and matched peritumoral samples were employed to analyze the RNF187 mRNA levels using qRT-PCR. Total RNA was extracted using the TRI Reagent (Invitrogen, USA) according to the manufacturer's protocol. Complementary DNA (cDNA) was synthesized from $2 \mu \mathrm{g}$ of total RNA using HieffTM First Strand cDNA Synthesis Super Mix (Yeasen, China). Amplification and detection were performed with $1 \mu \mathrm{l}$ cDNA and HieffTM qPCR SYBR ${ }^{\circledR}$ Green Master Mix (Yeasen, China). Glyceraldehyde 3-phosphate dehydrogenase (GAPDH) was used as the internal standard. Primers were RNF187: 5'-TGGAAATCATGAGAACTTG-3' and: 5'-ACGGTCCATCACGTGTCC-3'; GAPDH:
5'-GGCAT CCTGGGCTACACTGA-3' and 5'-GTGGTCGTTGAGGGCAATG-3'. The relative expression of RNF187 mRNA was analyzed by the comparative cycle threshold $(\mathrm{Ct})$. All experiments were repeated in triplicate.

\section{Western blot analysis and immunofluorescence}

Proteins extracted from HCC cells and samples were applied to detect RNF187 protein expression by western blotting as previously described [32]. The polyclonal rabbit anti-RNF187 (1:1000; Abcam, USA), polyclonal rabbit anti-JunD (1:500; Abcam, USA), Rat monoclonal to E-cadherin (1:5000; Abcam, USA), Rabbit polyclonal to snail (1:500; Abcam, USA), and Mouse monoclonal to Vimentin (1:1000; Abcam, USA) antibodies were used as the primary antibody, respectively. GAPDH (1:5,000; Chemicon, USA) was used as the internal control.

Polyclonal rabbit anti-human RNF187 (1:100, Abnova, China), rat monoclonal to E-cadherin (1:500), rabbit polyclonal to snail $(1: 100)$, and mouse monoclonal to Vimentin $(1: 100)$ antibodies were used for immunofluorescence. Nuclei were counterstained with 4', 6-diamidino-2-phenylindole (DAPI, Vector Laboratories, Burlingame, CA).

\section{Matrigel invasion, colony formation assay, and in vivo metastasis assays}

The matrigel invasion assay was carried out as follow. Briefly, $1 \times 10^{5}$ cells tumor cells suspended in Dulbecco's modified Eagle's medium (DMEM) / RPMI 1640 supplementing $0.1 \%$ fetal bovine serum (FBS) were added to the up chamber. The supernatant of containing NIH3T3 was added into the down chamber. After incubation for $48 \mathrm{~h}$, the invasive cells were counted under a Leica DMLA light microscope (Leica Microsystems, Wetzlar, Germany) as described [3]. The colony formation assay was performed as the previous report [28]. All experiments were carried out in triplicate.

$3 \times 10^{6} \mathrm{HCC}$ cells were suspended in $100 \mathrm{ml}$ DMEM and Matrigel (BD Biosciences, $1: 1$ ), and then inoculated into the liver parenchyma of nude mice after opening up the abdomen under anesthesia, and monitored once every 5 days for 45 days. The animals were killed under anesthesia and the visceral organs including the lungs and liver were examined by histopathological methods. Serial sections of mouse lungs were prepared and the number of lung metastases counted under a Leica DMLA light microscope (Leica Microsystems, Wetzlar, Germany) as the previous report [32].

\section{Construction of tissue microarrays (TMA) and immunohistochemistry}

Tissue microarrays were constructed as described in an earlier study [3]. Immunohistochemistry (IHC) 
was performed using a two-step detection system (Dako, Carpinteria, CA) as previously described. Briefly, formalinfixed and paraffin-embedded sections with a thickness of 4 $\mu \mathrm{m}$ were dewaxed in xylene and graded alcohols, hydrated, and washed in PBS. after microwave antigen retrieval (12 min in sodium citrate buffer, $\mathrm{pH} \mathrm{6),} \mathrm{the} \mathrm{endogenous}$ peroxidase was inhibited by $0.3 \% \mathrm{H}_{2} \mathrm{O}_{2}$ for $30 \mathrm{~min}$ and the sections were incubated with $10 \%$ normal goat serum for $30 \mathrm{~min}$. Primary antibodies (RNF187, 1:100) were applied overnight in a moist chamber at $4{ }^{\circ} \mathrm{C}$, followed by incubation for $30 \mathrm{~min}$ with the secondary antibody (RE7112, Novolink Polymer). The sections were developed in 3,3'-diaminobenzidine solution under microscopic observation and counterstained with hematoxylin. Negative control slides in which the primary antibodies omitted, were included in all IHC assays. The intensity of RNF187 was classified as high and low expressions (RNF187 $7^{\text {high }}>50 \%$ of tumor section, and RNF $187^{\text {low }} \leq 50 \%$, respectively).

\section{RNA sequence and analysis}

Total RNA was isolated from HCC cells and tissues using TRI Reagent (Invitrogen, USA) according to the supplier's protocol. The quality of RNA was evaluated using an Agilent 2100 Bioanalyzer (Agilent, Germany). cDNA synthesis was carried out according to the manufacturer's instructions, with the analysis performed by Majorbio (Shanghai, China). RNA-Seq reads were aligned to the human reference sequence National Center for Biotechnology Information (NCBI) hg19 with TopHat2 to calculate the values of fragments per kilobase of transcript per million mapped reads for known transcripts (FPKM). A two-class comparison of the pool of RNF187 high and the corresponding RNF187 low cell was performed to determine differentially expressed genes. We selected a fold change of 2 as the threshold for upregulation or downregulation, with a $P$-value $<0.05$ of the fold-change being considered to be statistically significant.

\section{Statistical analysis}

Statistical analyses were performed with SPSS 19.0 for Windows (Chicago, IL, USA) in this study. The RNF187 expression is described as means \pm standard deviations. The student's $t$-test was employed for comparison between groups of normally distributed data. The overall survival (OS) and disease-free survival (DFS) rates were assessed by the Kaplan-Meier analysis. A Cox's proportional hazards regression model was used to analyze the independent prognostic factors. A $P$-value $<0.05$ was considered to be statistically significant.

\section{Abbreviations}

HCC, hepatocellular carcinoma; EMT, epithelialmesenchymal transition; RNF187, ring finger protein
187; qRT-PCR, quantitative real-time polymerase chain reaction; cDNA, complementary DNA; shRNA, short hairpin RNA; FN1, fibronectin 1; MDM2, MDM2 protooncogene; BRCA1, breast cancer associated gene 1; CBLs, calcineurin B-like proteins; VHL, von HippelLindau tumor suppressor; TMA, tissue microarray; DFS, disease-free survival; OS, overall survival; AFP, alphafetoprotein; APC, anaphase promoting complex; KEGG, Kyoto encyclopedia of genes and genomes; $\mathrm{GO}$, gene ontology; FPKM, fragments per kilobase of transcript per million; CDC2, cyclin dependent kinase 1; HBEGF, heparin binding EGF like growth factor.

\section{Author contributions}

Conceived and designed the experiments: Y. P. X., Z. R. L. and X. L.Y.; Y. P. X., J.C.W. performed the experiments: Analysis of data: P. F. L., K. L., C. Y.: Contributed reagents/materials/analysis tools: Y.P.X., Z.R.L., J.C.W., and X.L.Y. Manuscript authors: Y.P.X., Z.R.L., and X .L.Y.

\section{CONFLICTS OF INTEREST}

Nothing to declare.

\section{FUNDING}

This work was supported by the National Natural Science Foundation of China (81260367, 81660489, 81160310 and 81071741).

\section{REFERENCES}

1. Torre LA, Bray F, Siegel RL, Ferlay J, Lortet-Tieulent J, Jemal A. Global cancer statistics, 2012. CA Cancer J Clin. 2015; 65:87-108.

2. Bruix J, Boix L, Sala M, Llovet JM. Focus on hepatocellular carcinoma. Cancer Cell. 2004; 5:215-219.

3. Ke AW, Shi GM, Zhou J, Wu FZ, Ding ZB, Hu MY, Xu Y, Song ZJ, Wang ZJ, Wu JC, Bai DS, Li JC, Liu KD, et al. Role of overexpression of CD151 and/or c-Met in predicting prognosis of hepatocellular carcinoma. Hepatology. 2009; 49:491-503.

4. Lee HE, Kim DH, Cho YH, Kim K, Chae SW, Sohn JH. Tumorto-tumor metastasis: Hepatocellular carcinoma metastatic to parathyroid adenoma. Pathol Int. 2011; 61:593-597.

5. Jou J, Diehl AM. Epithelial-mesenchymal transitions and hepatocarcinogenesis. J Clin Invest. 2010; 120:1031-1034.

6. Polyak K, Weinberg RA. Transitions between epithelial and mesenchymal states: acquisition of malignant and stem cell traits. Nat Rev Cancer. 2009; 9:265-273.

7. Lamouille S, Xu J, Derynck R. Molecular mechanisms of epithelial-mesenchymal transition. Nat Rev Mol Cell Bio. 2014; 15:178-196. 
8. Ke AW, Shi GM, Zhou J, Huang XY, Shi YH, Ding ZB, Wang XY, Devbhandari RP, Fan J. CD151 amplifies signaling by integrin alpha6betal to PI3K and induces the epithelial-mesenchymal transition in HCC cells. Gastroenterology. 2011; 140:1629-41.e15.

9. Grunert S, Jechlinger M, Beug H. Diverse cellular and molecular mechanisms contribute to epithelial plasticity and metastasis. Nat Rev Mol Cell Bio. 2003; 4:657-665.

10. Thiery JP, Sleeman JP. Complex networks orchestrate epithelial-mesenchymal transitions. Nat Rev Mol Cell Bio. 2006; 7:131-142.

11. Nieto MA. Epithelial plasticity: a common theme in embryonic and cancer cells. Science. 2013; 342:1234850.

12. Mani SA, Guo W, Liao MJ, Eaton EN, Ayyanan A, Zhou AY, Brooks M, Reinhard F, Zhang CC, Shipitsin M, Campbell LL, Polyak K, Brisken C, et al. The epithelialmesenchymal transition generates cells with properties of stem cells. Cell. 2008; 133:704-715.

13. Kudo-Saito C, Shirako H, Takeuchi T, Kawakami Y. Cancer metastasis is accelerated through immunosuppression during Snail-induced EMT of cancer cells. Cancer cell. 2009; 15:195-206.

14. Ravid T, Hochstrasser M. Diversity of degradation signals in the ubiquitin-proteasome system. Nat Rev Mol Cell Bio. 2008; 9:679-690.

15. Bedford L, Lowe J, Dick LR, Mayer RJ, Brownell JE. Ubiquitin-like protein conjugation and the ubiquitinproteasome system as drug targets. Nat Rev Drug Discov. $2011 ; 10: 29-46$.

16. Jiang JH, Liu YF, Ke AW, Gu FM, Yu Y, Dai Z, Gao Q, Shi GM, Liao BY, Xie YH, Fan J, Huang XW, Zhou J. Clinical significance of the ubiquitin ligase UBE3C in hepatocellular carcinoma revealed by exome sequencing. Hepatology. 2014; 59:2216-2227.

17. Cai JB, Shi GM, Dong ZR, Ke AW, Ma HH, Gao Q, Shen ZZ, Huang XY, Chen H, Yu DD, Liu LX, Zhang PF, Zhang $C$, et al. Ubiquitin-specific protease 7 accelerates p14(ARF) degradation by deubiquitinating thyroid hormone receptor-interacting protein 12 and promotes hepatocellular carcinoma progression. Hepatology. 2015; 61:1603-1614.

18. Davies CC, Chakraborty A, Cipriani F, Haigh K, Haigh JJ, Behrens A. Identification of a co-activator that links growth factor signalling to c-Jun/AP-1 activation. Nat Cell Biol. 2010; 12:963-972.

19. Davies CC, Chakraborty A, Diefenbacher ME, Skehel M, Behrens A. Arginine methylation of the c-Jun coactivator RACO-1 is required for c-Jun/AP-1 activation. EMBO J. 2013; 32:1556-1567.

20. Forster L, Finlayson J, Ghassemifar R. RNF187 is Downregulated Following NF-kappaB Inhibition in Late Erythroblasts. Biochem Genet. 2016; 54:714-721.

21. Snider NT, Omary MB. Post-translational modifications of intermediate filament proteins: mechanisms and functions. Nat Rev Mol Cell Bio. 2014; 15:163-177.
22. Beltrao P, Bork P, Krogan NJ, van Noort V. Evolution and functional cross-talk of protein post-translational modifications. Mol Systems Bio. 2013; 9:714.

23. Acconcia F, Sigismund S, Polo S. Ubiquitin in trafficking: the network at work. Exp Cell Res. 2009; 315:1610-1618.

24. Flemming A. Cancer: Opening the door to a new class of proteasome inhibitors. Nat Rev Drug Dis. 2012; 11:23.

25. Lipkowitz S, Weissman AM. RINGs of good and evil: RING finger ubiquitin ligases at the crossroads of tumour suppression and oncogenesis. Nat Rev Cancer. 2011; 11:629-643.

26. Berndsen CE, Wolberger C. New insights into ubiquitin E3 ligase mechanism. Nat Struct Mol Bio. 2014; 21:301-307.

27. Chakraborty A, Diefenbacher ME, Mylona A, Kassel O, Behrens A. The E3 ubiquitin ligase Trim7 mediates c-Jun/AP-1 activation by Ras signalling. Nat Commun. 2015; 6:6782.

28. Lukey MJ, Greene KS, Erickson JW, Wilson KF, Cerione RA. The oncogenic transcription factor c-Jun regulates glutaminase expression and sensitizes cells to glutaminasetargeted therapy. Nat Commun. 2016; 7:11321.

29. Trierweiler C, Hockenjos B, Zatloukal K, Thimme R, Blum HE, Wagner EF, Hasselblatt P. The transcription factor c-JUN/AP-1 promotes HBV-related liver tumorigenesis in mice. Cell Death Differ. 2016; 23:576-582.

30. Zhang P, Dong Z, Cai J, Zhang C, Shen Z, Ke A, Gao D, Fan J, Shi G. BRD4 promotes tumor growth and epithelialmesenchymal transition in hepatocellular carcinoma. Int $\mathrm{J}$ Immunopathol Pharmacol. 2015; 28:36-44.

31. Marijon H, Dokmak S, Paradis V, Zappa M, Bieche I, Bouattour M, Raymond E, Faivre S. Epithelial-tomesenchymal transition and acquired resistance to sunitinib in a patient with hepatocellular carcinoma. J Hepatol. 2011; 54:1073-1078.

32. Huang XY, Ke AW, Shi GM, Zhang X, Zhang C, Shi YH, Wang XY, Ding ZB, Xiao YS, Yan J, Qiu SJ, Fan J, Zhou J. alphaB-crystallin complexes with 14-3-3zeta to induce epithelial-mesenchymal transition and resistance to sorafenib in hepatocellular carcinoma. Hepatology. 2013; 57:2235-2247.

33. Schmitz KJ, Wohlschlaeger J, Lang H, Sotiropoulos GC, Malago M, Steveling K, Reis H, Cicinnati VR, Schmid KW, Baba HA. Activation of the ERK and AKT signalling pathway predicts poor prognosis in hepatocellular carcinoma and ERK activation in cancer tissue is associated with hepatitis C virus infection. J Hepatol. 2008; 48:83-90.

34. Shi GM, Ke AW, Zhou J, Wang XY, Xu Y, Ding ZB, Devbhandari RP, Huang XY, Qiu SJ, Shi YH, Dai Z, Yang $\mathrm{XR}$, Yang GH, et al. CD151 modulates expression of matrix metalloproteinase 9 and promotes neoangiogenesis and progression of hepatocellular carcinoma. Hepatology. 2010; 52:183-196. 